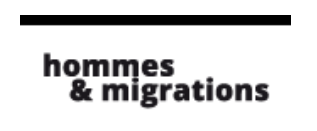

Hommes \& migrations

Revue française de référence sur les dynamiques

migratoires

1308 | 2014

Les Paris des migrants

\title{
Les quartiers africains de Paris comme lieux d'inclusion et d'exclusion par la consommation
}

\section{Virginie Silhouette-Dercourt}

\section{Q OpenEdition}

Journals

\section{Édition électronique}

URL : http://journals.openedition.org/hommesmigrations/3004

DOI : 10.4000/hommesmigrations.3004

ISSN : 2262-3353

\section{Éditeur}

Musée national de l'histoire de l'immigration

\section{Édition imprimée}

Date de publication : 1 octobre 2014

Pagination : 97-103

ISBN : 978-2-919040-29-2

ISSN : $1142-852 X$

\section{Référence électronique}

Virginie Silhouette-Dercourt, «Les quartiers africains de Paris comme lieux d'inclusion et d'exclusion par la consommation », Hommes \& migrations [En ligne], 1308 | 2014, mis en ligne le 01 octobre 2017 consulté le 01 mai 2019. URL : http://journals.openedition.org/hommesmigrations/3004 ; DOI :

10.4000/hommesmigrations.3004 


\title{
LES QUARTIERS AFRICAINS DE PARIS COMME LIEUX D'INCLUSION ET D'EXCLUSION PAR LA CONSOMMATION
}

par VIRGINIE SILHOUETTE-DERCOURT, maître de conférences, université Paris-XIII-CEPN, Centre Marc-Bloch, Berlin.

\author{
À Paris, certains lieux de consommation sont presque \\ entièrement dédiés aux populations originaires d'Afrique \\ subsaharienne. Circonscrits autour de deux stations de métro \\ emblématiques, Château-d'Eau et Château-Rouge, ces quartiers \\ constituent des pôles commerciaux où abondent les produits \\ venus de toute I'Afrique. De quoi satisfaire les besoins \\ d'une clientèle "ethnique" en cosmétiques et biens alimentaires. \\ Mais derrière les étals colorés court une invisible frontière: \\ celle de la gentrification urbaine qui peu à peu gagne le nord \\ de la capitale.
}

Alors que la crise économique et l'afflux de réfugiés victimes de conflits interrogent les modèles d'intégration européens, se pose pour les migrants et leurs enfants la question de leur survie économique. Dans les capitales européennes, des entreprises, des commerces, des marchés, des restaurants ou des centres commerciaux se développent, portés par ces populations qui emploient du personnel et vendent des marchandises issues de filières transnationales ${ }^{1}$. Ces espaces de consommation, enclavés au sein de métropoles en cours de gentrification, sont des lieux extrêmement actifs qui facilitent l'intégration. Ce sont aussi des espaces d'échanges sociaux et culturels "entre soi" qui passent par la consommation de produits du pays. Ce sont enfin des lieux où s'expriment de façon quotidienne les rapports de pouvoir avec l'État. Une enquête ethnographique ${ }^{2}$ dans deux quartiers du nord de Paris - Château-Rouge et Château-d'Eau - tend à montrer que la consommation qui s'y déploie reflète ces dynamiques contradictoires de ressourcement identitaire et de métissage culturel, de construction de frontières et de dilution dans la société française. 


\section{Des centres de consommation africains extrêmement actifs}

En 2011, on comptait en France 5, 6 millions d'immigrés ${ }^{3}$, dont près de $13 \%$ en provenance d'Afrique subsaharienne $e^{4}$. Cette proportion est en progression depuis quelques années ${ }^{5}$ mais reste modeste par rapport à celle des immigrés en provenance d'Afrique du Nord (29,6 \%) ou d'Europe (37,1 \%). Ces chiffres n'incluent pas les descendants directs d'immigrés, c'est-à-dire les personnes nées en France et ayant au moins un À Paris justement, parent immigré. En 2008,

où l'activité commerciale se développe principalement dans

les quartiers centraux,

il existe des pôles d'attraction

spécialisés à destination de la population d'origine africaine, notamment autour des stations de métro Château-d'Eau et Château-Rouge.

destination de la population d'origine africaine, notamment autour des stations de métro Château-d'Eau et Château-Rouge ${ }^{9}$. À partir des années 1960 à Paris, se développe dans quelques épiceries une offre de produits alimentaires "exotiques" pour répondre aux besoins d'une population immi- grée croissante ${ }^{10}$. Un peu plus tard, d'autres boutiques ouvrent dans le nord de Paris (XVIII et XX'). À partir des années 1980, le nombre de commerces augmente très fortement et se concentre dans certaines zones spécialisées comme les quartiers de Château-Rouge et de Château-d'Eau, où réside traditionnellement une importante population immigrée. Si la pression immobilière a poussé une part grandissante des populations immigrées en dehors de ces quartiers, elles y reviennent pour trouver les produits et l'ambiance du pays.

\section{La beauté afro}

Le quartier de Château-d'Eau ${ }^{12}$ a une surface commerciale bien moindre que celle de ChâteauRouge (un peu moins de $1 \mathrm{~km}$ de façade commerciale totale). De plus, à la différence de ChâteauRouge, la quasi-totalité de son activité commerciale "exotique" est spécialisée dans la beauté afro. On remarque également quelques commerces de téléphonie mobile, des restaurants africains, un magasin de produits culturels et des agences de transfert d'argent. Un peu plus de 100 commerces proposent pour moitié de la coiffure, et pour l'autre moitié des cosmétiques, des mèches, des tresses et des perruques. Ces boutiques sont en majorité de toutes petites échoppes de quelques mètres carrés, parfois des surfaces plus importantes, avec une partie de la

\footnotetext{
3. Selon la définition adoptée par le Haut Conseil à l'intégration, un immigré est une personne née étrangère à l'étranger et résidant en France. Un descendant direct d'immigré est une personne née en France métropolitaine d'au moins un parent né étranger à l'étranger. 4. Selon le recensement Insee de 2011. 5. L'immigration subsaharienne a contribué à raison d'un quart à l'accroissement de l'ensemble de la population immigrée constaté entre 1999 et 2008. Voir Gérard Bouvier, Immigrés et descendants d'immigrés en France, Paris, Insee, 2012. 6. Ibid. 7. Catherine Borrel, Bertrand Lhommeau, “Être né en France d'un parent immigré", in Insee première, $n^{\circ} 1287$, mars $_{2010 .}$

8. Entre 2007 et 2011, sur environ 84000 locaux recensés en pied d'immeuble, 73 \% sont occupés par une activité commerciale. À Paris, l'activité commerciale est dominée par les commerces non alimentaires (1 sur 3), alors que les commerces alimentaires représentent un commerce sur 10. Parmi les commerces non alimentaires, ceux qui se consacrent aux soins de beauté et autres soins corporels connaissent un fort développement - respectivement $+4,4 \%$ par an sur la période $2007-2011$ et $21 \%$ pour les soins corporels (Source : L'Évolution des commerces à Paris : inventaire des commerces 2011 et évolutions 2007-2011, Atelier parisien d'urbanisme, Mairie de Paris, Chambre de commerce et d'industrie de Paris, janvier 2012). 9. Autre exemples : les commerces dans le "Triangle de Choisy" (qui se prolonge sur la Dalle des Olympiades) dans le XIII arrondissement ou autour de la station Belleville attirent une clientèle asiatique, tandis qu'on trouve une concentration de commerces s'adressant plus particulièrement à la diaspora tamoule srilankaise autour de la station de métro La Chapelle (ligne 2). Le marché de Saint-Denis (93) est également un lieu d'achat particulièrement prisé par la population d'origine africaine. 10. Voir Honoré Tabuna, Évaluation des échanges des produits forestiers non ligneux entre l'Afrique subsaharienne et l'Europe, Organisation des Nations unies pour l'alimentation et l'agriculture, Bureau central pour l'Afrique, FAO, 2000. 11. Atelier parisien d'urbanisme (Apur), Commerce et espace public à Château-Rouge, fiche synthétique, Mairie de Paris, Chambre de commerce et d'industrie de Paris, 2004. 12. Le quartier de Château-d'Eau s'étend sur le boulevard de Strasbourg entre la gare de l'Est au nord et le boulevard de Saint-Denis au sud, ainsi que sur la rue de Château-d'Eau (autour de la station de métro).
} 
boutique consacrée aux mèches ou aux perruques, et l'autre aux produits cosmétiques. Enfin, un troisième groupe de commerces (environ 20 points de vente de taille plus grande), situés au milieu et en bas du boulevard de Strasbourg, revendique l'appellation “institut de beauté", comme HT26, MGC, Makari, Fair \& White, QUEI+, Françoise Bedon ou Patricia Reynier. Certains de ces commerces sont extrêmement actifs avec une clientèle nombreuse. À la sortie du métro Château-d'Eau et devant les boutiques, de nombreux rabatteurs attirent les clientes vers les salons de coiffure ou les boutiques. Des vendeurs à la sauvette proposent, sur de minuscules étals, des fruits et légumes à côté de préparations cosmétiques pour éclaircir la peau...

\section{L'offre de produits "exotiques"}

Dans le même temps, l'offre de produits "exotiques", essentiellement concentrée dans ces quartiers et destinée à une population africaine, s'est diffusée à partir des années 1990 en grande distribution sous une forme "marketée". Inspirés par leurs succès sur les "ethnic markets" états-uniens et en s'appuyant sur ce qui se passe dans les quartiers "exotiques", des groupes internationaux ont commencé à proposer des offres en grande distribution à la fois dans ces quartiers et aussi en dehors. Dans le quartier de Château-d'Eau, par exemple, Monoprix a deux magasins (l'un en haut de la rue du Fau-
bourg-Saint-Denis et l'autre sur le boulevard SaintDenis), tandis que Franprix s'est installé boulevard de Magenta. Ces magasins proposent un rayon de produits "exotiques", à la fois dans le domaine alimentaire et dans celui de l'hygiène-beauté.

De même, hors des quartiers de Château-d'Eau et Château-Rouge, les grands groupes de distribution en France comme Carrefour, Leclerc, Auchan ou Casino ont développé des offres ${ }^{13}$ et de l'espace dans les linéaires pour tirer profit de la dynamique de ces segments ${ }^{14}$. Il est vrai qu'aujourd'hui les marchés 'ethniques' - que ce soit les produits halal ${ }^{15}$, la mode vestimentaire ou capillaire, les cosmétiques ${ }^{16}$ ou la téléphonie mobile ${ }^{17}$ et les services de transfert d'argent ${ }^{18}$ - croissent rapidement en France et ces offres n'intéressent plus seulement les communautés d'immigrés mais aussi le consommateur "de souche" en quête d'authenticité et de nouvelles expériences. Cependant, alors que la grande distribution en France contrôle plus de 60 \% des ventes totales du marché alimentaire ${ }^{19}$, entre 70 et $80 \%$ des ventes "exotiques" restent toujours réalisées dans les petits magasins traditionnels ${ }^{20}$ dans des quartiers comme Château-Rouge et Château-d'Eau.

\section{Des lieux de consommation qui facilitent l'intégration sociale}

Ces deux quartiers sont bien souvent les premiers endroits où l'on vient après être arrivé sur le territoire : on y retrouve des amis, de la famille, tout 
un réseau d'entraide qui va faciliter les premiers pas dans la société française. En cas de difficultés (chômage, soucis familiaux), ce réseau d'entraide pourra être réactivé et fournir de nouvelles perspectives économiques, donner des conseils pour l'éducation en France des enfants, sur le système de santé...

Ce sont aussi des lieux de ressourcement culturel. On y trouve des produits du pays adaptés à ses goûts et à ses besoins. "Château-Rouge, c'est l'Afrique pour moi... les produits que j'y trouve, c'est pareil, tout est importé... alors que Monoprix, les produits ont été fabriqués, peut-être pas en France mais dans des usines occidentales." De même pour les produits vendus dans les magasins de ces quartiers: "Le magasin est physiquement là, mais les produits ne sont pas d'ici, tout est importé." Pendant les fêtes culturelles ou religieuses, il est possible d'acheter en quantité suffisante, et à un prix tout à fait raisonnable, les ingrédients

Ces deux quartiers sont bien souvent les premiers endroits où l'on vient après être arrivé sur le territoire on y retrouve des amis, de la famille, tout un réseau d'entraide qui va faciliter les premiers pas dans la société française. ou les produits pour les soins du visage: "Ce que je recherche vraiment à Château-d'Eau et que je n'arrive pas à trouver en grandes surfaces, ce sont les produits pour le visage... pour les taches noires, les boutons." Parfois, les prix pratiqués près de chez elles sont trop élevés. Elles se rendent alors dans ces quartiers qu'elles associent à "là-bas", au pays, pour trouver les produits qui leur conviennent vraiment, et à un prix plus faible. D'après elles, cela revient aussi moins cher d'aller à Château-Rouge ou à Châteaud'Eau : "Là, par exemple, mon fond de teint, j'en ai eu pour 13 euros, mais si je vais chez Sephora, jen ai pour 30 ou 40 euros, donc je gagne au moins 15 ou 20 euros, c'est pas négligeable." Dans le domaine de la coiffure, il est très souvent difficile de trouver près du domicile un coiffeur sachant travailler le cheveu africain : "Je vais dans le centre commercial à côté de chez moi, il $y$ a des coiffeurs l'un après l'autre, mais aucun ne s'intéresse à mon cheveu, ils ne savent pas coiffer le cheveu africain." Les femmes ont souvent recours aux services d'une proche (sœur, cousine, amie) avec laquelle elles discutent de la coiffure. Elles se renseignent sur Internet sur les tendances en matière de beauté du cheveu en Afrique, puis se rendent à Château-d'Eau pour acheter les éléments nécessaires (mèches, postiches, perruques, lace wigs...), avant de passer plusieurs heures - voire plusieurs jours - à la confection de la coiffure (lissage, bouclage, tissage, pose d'extensions...). Autre solution : elles se rendent dans le quartier de Château-d'Eau pour trouver à un prix modique un coiffeur qui saura élaborer cette coiffure.

\section{Des supports pour la négociation des identités}

D'autres dimensions plus identitaires émergent des discours des personnes fréquentant les deux quartiers. Parfois, elles se sentent totalement insérées dans la société française et suivent les mêmes parcours d'achat que "tout le monde", mais parfois aussi elles ne se sentent pas tout à fait d'ici et suivent du coup d'autres parcours, qu'elles associent davantage à leur pays d'origine : "Nous, on s'est habituées aux produits français. Mais parfois on a envie de retrouver des choses du pays, de faire comme ils font au pays". Dans ces moments-là, la fréquentation de ces quartiers permet de retrouver cette partie de l'identité associée au pays d'origine: "Quand tu as le blues du pays et que tu veux rencontrer du monde, des copines que tu n'as pas vues depuis longtemps, tu vas à Château-Rouge." Cette dimension des comportements est très présente. "Quand, pendant deux semaines, on n'a pas mangé quelque chose du pays, ça se manifeste." Au cours de l'année, certains moments vont déclencher le besoin de se sentir "comme au pays". La nos- 
talgie de la terre natale peut être fonction des saisons :"Des fois je me lève et ça me manque. Je vois le temps et je me dis que je me verrais bien au pays. Même au niveau de la nourriture, un bon plat, les mêmes goûts que là-bas." Parfois, le déclencheur, ce sont les fêtes religieuses ou culturelles qui ont lieu au pays : "Ils vont se retrouver, je ne serai pas là avec eux." Parfois aussi, surviennent des expériences sociales négatives vécues dans le quotidien, ou encore le rejet de comportements associés à la culture française. Pendant ces moments particuliers, rechercher des images du pays sur Internet, appeler la famille restée sur place, y faire des plans de voyage ou écouter de la musique du pays permet d'apaiser le mal-être
: "Ce sont des périodes où je vais me remettre à écouter de la musique de là-bas, alors que je ne le fais jamais d'habitude, je regarde les billets d'avion et je me dis allez hop, je pars." Tout comme aller se promener avec des ami(e)s à Château-Rouge ou à Château-d'Eau pour y acheter des ingrédients du pays et réactiver un rituel de beauté : "L'hiver, quand il fait trop froid, je prends une douche très chaude et je m'enduis le corps de beurre de karité, après, ça va mieux." Ce retour vers des éléments de la culture d'origine qui passe par la consommation nourrit le sentiment d'appartenance au pays et permet d'aller de l'avant. Certaines personnes, ici un gérant de boutique de produits cosmétiques, expriment cet attachement au quar- 
tier de façon assez dramatique: "Sans ce quartier (Château-Rouge), on ne pourrait pas vivre ici, ce serait le suicide..."

Du coup, les personnes interrogées font une claire distinction entre les quartiers et les commerces qui répondent aux besoins des "gens d'ici" et ceux qui répondent aux besoins des "gens de là-bas". Par exemple, Monoprix ou Auchan sont, dans les discours, exclusivement associés à "ici". D'autres sont exclusivement associés à "là-bas", comme les quartiers de Château-d'Eau et de Château-Rouge. Ces quartiers sont plus à même de répondre à leur besoin d'appartenance.

Cependant, elles émettent également de fortes critiques sur ces quartiers, ells expriment parfois une forme de rejet. Notamment en ce qui concerne les produits cosmétiques, elles se posent des questions sur leurs lieu et conditions de fabrication: "Je n'ai aucune idée d'où ils viennent et depuis combien de temps les produits sont là, dans la boutique." Elles citent des cas de cosmétiques vendus sous une marque reconnue mais ayant causé des problèmes de tolérance, ou de dates de péremption non indiquées sur les produits: "On ne sait pas dans quelles conditions ils sont arrivés là ; ce sont quand même un peu des marchés parallèles." Elles sont conscientes que ces zones n'offrent pas toujours le même niveau de garantie en terme de sécurité que les produits achetés dans les magasins occidentaux. "Moi, je vais toujours dans la même boutique, là je sais qu’ils sont sérieux."

\section{Construction et théâtralisation des frontières}

Les rapports de pouvoir entre les autorités de l'État et les personnes présentes dans ces quartiers de consommation "exotique" font partie du quotidien. La surveillance par des représentants des forces de l'ordre habillés en civil y est palpable. À la sortie du métro Château-Rouge, à côté des boutiques sédentaires, les activités de vente à la sauvette de téléphones portables, de mélanges cosmétiques dépigmentants ${ }^{21}$ (parfois à l'hydroquinone, donc interdits), de bagagerie de contrefaçon, de fruits et légumes... sont particulièrement visibles.

Pour les migrants sans papiers fraîchement arrivés, ces petites activités de commerce ou l'emploi non déclaré dans une des boutiques permettent de survivre les premiers mois. Mais les saisies de la police, qui évolue dans ces quartiers, sont fréquentes et punissent les contrevenants.

Pendant mon enquête sur le terrain en 2013, j'ai assisté à plusieurs interventions pour saisir des marchandises de contrefaçon. Ces interventions soudaines déclenchent des courses poursuites et des mouvements de foule, les autres vendeurs à la sauvette tentant d'échapper aux forces de l'ordre. Quelques minutes plus tard, ils reprennent position au même endroit jusqu'à la prochaine fois. Il existe du coup une sorte de loi du silence des commerçants qui ne veulent pas toujours témoigner. Le personnel présent en boutique se retranche derrière un gérant de magasin, non présent sur les lieux.

Ce personnel n'a pas toujours les papiers nécessaires, ce qui peut entraîner des pratiques abusives en terme de droit du travail (absence de contrat, retard de paiement ou non paiement des heures travaillées... $)^{22}$.

En 2012, le quartier de Château-Rouge est devenu une zone de sécurité prioritaire (ZSP), après celui de Saint-Denis et de Saint-Ouen (93). Selon les Cahiers de la Préfecture de police (2012), les ZSP "ont pour vocation de renforcer la

\footnotetext{
21. La dépigmentation de la peau est une pratique encore courante dans les communautés noires. Elle touche à la fois les femmes, les hommes et aussi parfois les enfants. Elle s'effectue à l'aide de mélanges de crèmes contenant des matières nocives (hydroquinone, dermocorticoïdes, mercure...) interdites à la vente sous forme de cosmétiques. Les quartiers de Château-Rouge et de Château-d’Eau restent des lieux de vente de ces produits illicites malgré les campagnes de sensibilisation menées par la Mairie de Paris en collaboration avec l'Agence de contrôle des produits sanitaires (AFSSAPS) et la Direction générale de la concurrence, de la consommation et de la répression des fraudes (DGCCRF). 22. Voir, par exemple, le cas des 7 salariés sans papiers du salon de coiffure Supply Beauty dans le quartier de Château-d'Eau, qui se sont mis en grève en février 2014. Ils n'avaient pas perçu de salaires depuis deux mois et effectuaient des journées de treize heures dans des locaux insalubres.
} 
sécurisation de certains territoires particulièrement touchés par une dégradation de l'ordre et de la tranquillité publics".

Ce dispositif, qui repose sur une synergie entre forces de sécurité intérieure, autres services de l'État (justice, douanes, finances publiques et Éducation nationale), collectivités locales et partenaires de l'arrondissement, a pour objectif de "réduire les actes de délinquance ou d'incivilité : ventes à la sauvette, prostitution et racolage, consommation d'alcool sur la voie publique, le non-respect des règles d'hygiène et de sécurité, le non-respect des règles municipales liées à l'occupation de la voie publique". Depuis cette date, la présence des policiers en tenue et en civil s'est encore accrue dans ce quartier de jour comme de nuit et crée une sorte de tension et d'état d'alerte permanent.

Ces dispositifs de surveillance et de punition, omniprésents et théâtralisés dans le quotidien de ces quartiers de consommation, renvoient à d'autres dispositifs d'exclusion érigés aux frontières de l'Union européenne. Ils prolongent cet effort permanent de construction et de colmatage de frontières entre "eux" et "nous".

\section{Conclusion}

À Château-Rouge et à Château-d'Eau se tissent jour après jour des liens transnationaux entre entrepreneurs issus de l'immigration et réseaux économiques dans les pays d'origine. Ces liens, parfois très anciens, continuent de se développer malgré le contrôle, la limitation et le management général des flux migratoires aux frontières de l'Union européenne et de la France. Les quartiers de consommation "exotiques" sont des lieux de création, où migrants et descendants de migrants nourrissent, renouvellent, élargissent les codes et les pratiques de consommation nationaux. Par la mise à disposition d'objets issus "d'ailleurs", ces quartiers stimulent l'hybridation et la créolisation culturelles, ainsi que l'émergence de nouvelles esthétiques qui se diffusent ensuite hors des quartiers, dans d'autres lieux de consommation. Malgré les fermetures et les rapports de pouvoir qui s'y développent, ils sont une sorte de fabrique quotidienne d'une France multiculturelle ouverte sur le monde. 